arbeiteten quantitativen Verhältnisse eine verstärkte oder verminderte Betaninausscheidung durch allergische Erscheinungen verursacht sein kann, läßt sich noch nicht sagen. Genetische Faktoren scheinen keine Rolle zu spielen. Ebensowenig ist für die Betaninausscheidung ein stark saurer Urin Voraussetzung; dagegen kann in einem stärker alkalischen Urin Betanin zerstört werden. Eine Fermentadaptation als Ursache für einen schnellen Abbau resorbierten Betanins, wodurch das Erscheinungs- bild des „Nichtausscheiders“ entstehen könnte, scheint, wie aus unseren Langzeitversuchen hervorgeht, keine Rolle zu spielen. Im Serum konnten wir nach Rote Rüben-Aufnahme und Sicherung der Resorption von Betanin durch Nachweis von Betanin im Harn Betanin nicht nachweisen, damit lag die Konzentration sicher unter $300 \mu \mathrm{g} \%$. Frühere Arbeitsergebnisse erscheinen unsicher, da ein Hämoglobingehalt des Serums Betanin vortäuschen kann.

\title{
Literatur
}

1. Wilcox, M. E., H. Wyler und A. S. Dreiding, Zur Konstitution des Randenfarbstoffes Betanin. 8. Mitteilung: Stereochemie von Betanidin und Isobetanidin. Helv. Chim. Acta 48, 1134 (1965). - 2. Schudel, G., Úber die Anthocyane von Beta vulgaris L. (var. rapacea Koch forma rubra L.) und Raphanus sativus L. (var. Radicula Pers.). Dissertation Zürich (1918). 3. Aintey, A.D. und R. Robinson, Nitrogenous anthocyanins. Part III. Preliminary experiments with betanidin. J. Chem. Soc. (London) 1937, 446. - 4. Pucher, G.W., L. C. Curtis und H. B. VICKERY, The red pigment of the root of the beet (Beta vulgaris). I. The preparation of betanin. J. Biol. Chem. 123, 61 (1938). - 5. Pucher, G. W., L. C. Curtis und H. B. Vickery, The red pigment of the root of the beet (Beta vulgaris). II. A method to determine betanin. J. Biol. Chem. 123, 71 (1938). 6. ReZnIK, H., Dic Pigmente der Centrospermen als systematisches Element II. - Untersuchungen über das ionophoretische Verhalten. Planta 49, 406 (1957). - 7. WyLER, H., G. Vincentr, M. Mercier, G. Sassu und A. S. Dreiding, Zur Konstitution des Randenfarbstoffes Betanin, 2. (vorläufige) Mitteilung. Helv. Chim. Acta 42, 1696 (1959). - 8. Schmidt, O. Th., P. Becher und M. HüBneR, Zur Kenntnis der Farbstoffe der Roten Rübe, III. Chem. Bericht 93, 1296 (1960). - 9. GeldmaCher-v. MaLLINCKRoDt, M. und K. MENDNER, Die Betaninurie: Nachweis und gerichtsmedizinische Bedeutung. Dtsch. Z. ges. gerichtl. Med. 56, 287 (1965). - 10. Arello, M.-Th., Zur Genetik der Betaninurie. Dissertation Erlangen (1965). - 11. HEss, A. F. und V. C. Myers, Carotinemia: A new clinical picture. J. Amer. Med. Assoc. 73, 1743 (1919). - 12. RuH, H. O. und J. A. Garvin, Anthocyaninemia and anthocyaninuria. Ohio State Med. J. 20, 763 (1924). - 13. Cathala, J., P. Martrou und L. Gras, Sur le passage du pigment de la betterave rouge dans les urines. Bull. Soc. Pédiat. de Patis 37, 88 (1939). - 14. Marheson, A., Anthocyaninuria. Am. J. Dis. Child. 51, 226 (1936). - 15. HALLÉ, J. und L. Girard, Passage du pigment de la betterave rouge dans l'urine de l'enfant. Bull. Soc. Pédiat. de Paris 36, 266 (1938). 16. JACOBSEN, E., Rød urin efter spisning af rødbeder. Ugeskrift Laeg. (Dän.) 104, 136 (1942). - 17. Allison, A. C. und K. G. MCWHIRTER, Two unifactorial characters for which man is polymorphic. Nature 178, 748 (1956). - 18. Saldanha, P. H., L. E. Magalhaes und W. A. Horta, Race differences in the ability to excrete beetroot pigment (Betanin). Nature (Lond.) 187, 806 (1960). - 19. Saldanha, P. H., O. Frota-Pessoa und L. I. S. Perxoro, Genetik der Betanin-Ausscheidung bei holländischen Einwanderern in Brasilien. In: Second International Conference of Human Genetics, Roma, D. 221 (1961). - 20. JeRvrs, J. J., Acidosis and hepatic disorder. Brit. Med. J. 1, 1408 (1914). 21. Poole, M. W., Anthocyaninuria. Amer. J. Dis. Child. 33, 784 (1927). - 22. LaUR, A., Erythrurie nach Rotrübengenuß. Klinisch-experimenteller Beitrag zum Problem der Darmresorption. Dtsch. Arch. Klin. Med. 196, 466 (1949). - 23. ZindLer, G. A. und G.C. Colovos, Anthocyaninuria and beet allergy. Ann. Allergy 8, 603 (1950). - 24. Luke, R. G. und W. C. Watson, Anaphylaxis with beeturia. Brit. Med. J. 1963, 980. - 25. Watson, W. C., R. G. Luke und J.A. INALL, Beeturia, Its incidence and a clue to its mechanism. Brit. Med. J. 1963, 971 . 26. Mendiner, K., Die Betaninurie, Nachweis und gerichtsmedizinische Bedeutung. Dissertation Erlangen (1965). - 27. Arelro, M. V., Untersuchungen zum qualitativen Nachweis und zur quantitativen Bestimmung von freiem Betanin im Serum. Dissertation Erlangen (1964).

Priv.-Doz. Dr. Dr. M. Geldmacher-v. Mallinckrodt 852 Erlangen, Universitätsstr. 22

\section{Ein optischer Test zur Bestimmung der Kreatin-Phosphokinase}

\author{
Von M. Feraudi und K. Harm \\ Aus dem Pbysiologisch-Chemischen Institut der Universität Hamburg-Eppendorf (Direktor: Prof. Dr. J. Kühnau) \\ (Eingegangen am 4. Februar 1967)
}

Es wird die Zusammensetzung eines optischen Tests zur Bestimmung der CPK (2. 7.3.2 ATP: Kreatin-Phosphotransferase) nach dem Reaktionsschema von M. L. Tanzer und CH. GrLvarg angegeben. Durch die Ermittlung optimaler Reaktionsbedingungen hat der Test eine wesentlich höhere Empfindlichkeit als die bisher bekannten Verfahren. Die gemessene Aktivität ist der Enzymkonzentration in der Küvette proportional, und zwar bei Einsatz von kristalliner CPK im Bereich von 0,06 bis $0,004 \mu \mathrm{Mol} /(\mathrm{Min}$. $\cdot \mathrm{ml})$ und bei Einsatz von menschlichem Serum im Bereich von 0,005 bis $0,0001 \mu \mathrm{Mol} /(\mathrm{Min} . \cdot \mathrm{m} l)$.

A procedure is described for the coupled photometric measurement of CPK (2.7.3.2 ATP: creatine phosphotransferase) according to the reaction mechanism of M. L. TANZER and CH. GrLvarg. By establishing optimal reaction conditions the sensivity of the method was markely increased. The activity level measured is proportional to the concentration of enzyme in the cuvettes for crystallized CPK in the range $0,06-0,004 \mu \mathrm{mol} /(\mathrm{min} \cdot \mathrm{m} l)$ and for human serum in the range $0,005-0,0001 \mu \mathrm{mol} /(\mathrm{min} \cdot \mathrm{m} l)$.

Es gibt verschiedene Verfahren $(1,2,3,4,5)$, um die Aktivität der CPK (2.7.3.2-ATP: Kreatin-Phospho- transferase) im optischen Test zu messen. Jèdoch fehlen Angaben darüber, wie die Zusammensetzung der ein- 
Tab. 1

Bestimmung der Adenosin-5'-triphosphat: Kreatin-Phosphotransferase. Meßtemperatur 38,0-40,0 $0^{\circ}$ Alle Reagenzien sind in Pufferlösung gelöst. Es werden $0,050 \mathrm{~m} l$ des unten angegebenen Testansatzes in eine Küvette der Schichtdicke $1,000 \mathrm{~cm}$ und der lichten Breite $2 \mathrm{~mm}$ einpipettiert

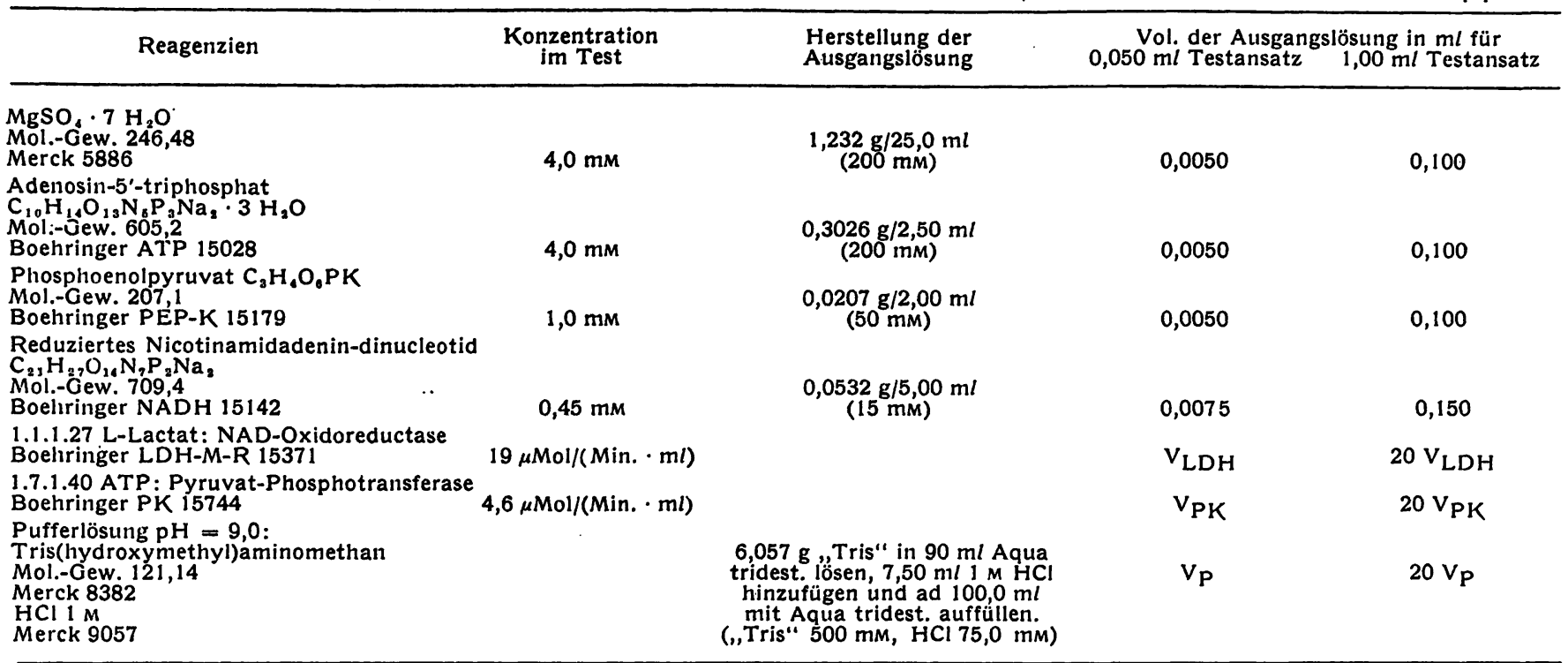

Wenn die Meßtemperatur erreicht ist, werden $0,075 \mathrm{ml}$ der zu testenden Lösung hinzugefügt und die Reaktion mit $0,125 \mathrm{ml} \mathrm{Kreatinlösung}$ (Merck 5205; $150 \mathrm{rm}$ : 0,0 $83 \mathrm{~g}$ mit Pufferlösung ad $5,00 \mathrm{ml}$ ) gestartet, nachdem vorher mittels eines PVC-Fadens gut durchgemischt wurde. $\mathrm{Da}$ Kreatin selır scliwer löslich ist, empfiehlt es sich, die Kristalle gut $\mathbf{z u}$ mörsern. Bei einer Temperatur von $1-4^{\circ}$ sind die Ausgangslösungen eine Woche lang haltbar. Die Kreatinlösung wird kurz vor der Bestimmung hergestellt. Da die Enzym-Präparate oft nicht die vom Hersteller angegebene Aktivität aufweisen, ist es erforderljch, jeweils vor Benutzung einer neuen Suspension deren Aktivität $[\mu M o l /(M i n$. · ml)] festzustellen und danach die Menge der Hilfsenzyme im Testansatz zu berechnen. Die Testansätze für die Messung der Aktivität der Hilfsenzyme sind in den folgenden Tabellen angegeben.

Tab. 2

Bestimmung der L-Lactat: NAD-Oxidoreductase. Meßtemperatur $(48,0+0,2)^{\circ}$. Alle Reagenzien sind in Aqua tridest. gelöst. Es werden 1,93 ml des unten angegebenen Testansatzes in eine Küvette der Schichtdicke $1,000 \mathrm{~cm}$ einpipettiert

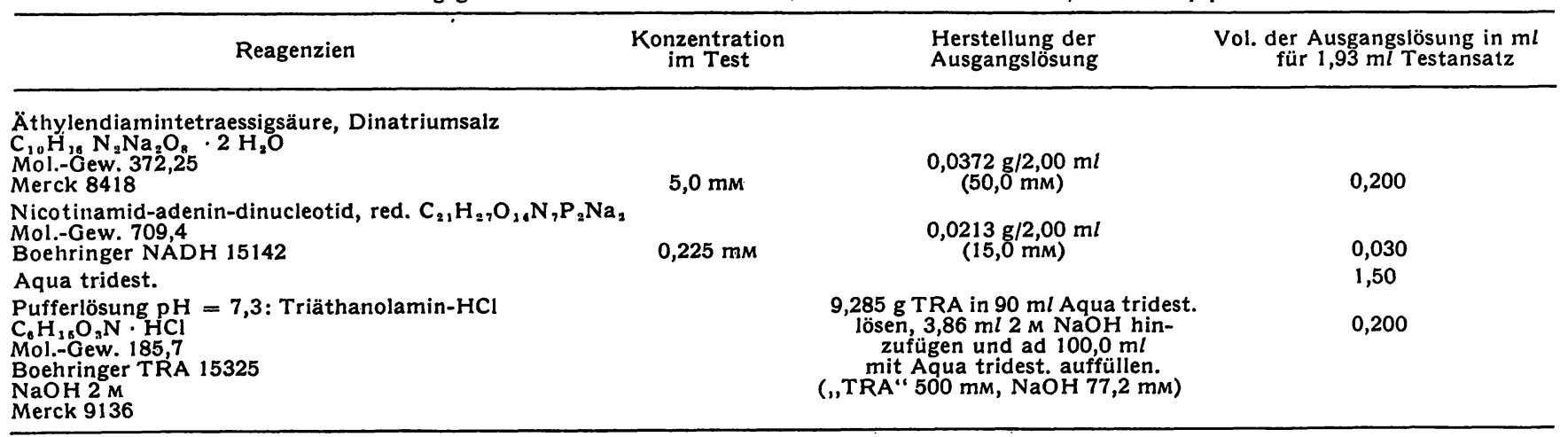

Wenn die Meßtemperatur erreicht ist, werden $0,050 \mathrm{ml}$ der zu testenden Lösung $\left(2 \cdot 10^{-3}\right.$ Verdünnung der Enzym-Suspension, die unmittelbar

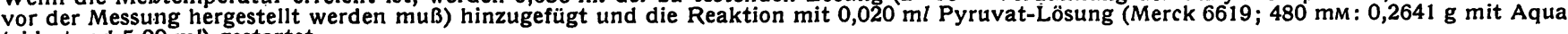
tridest. ad $5,00 \mathrm{ml}$ ) gestartet.

Tab. 3

Bestimmung der ATP:Pyruvat-Phosphotransferase. Meßtemperatur $(38,0 \pm 0,2)^{\circ}$. Alle Reagenzien sind in Pufferlösung gelöst. Es werden $1,75 \mathrm{ml}$ des unten angegebenen Testansatzes in eine Küvette der Schichtdicke $1,000 \mathrm{~cm}$ einpipettiert

\begin{tabular}{|c|c|c|c|}
\hline Reagenzien & $\begin{array}{l}\text { Konzentration } \\
\text { im Test }\end{array}$ & $\begin{array}{l}\text { Herstellung der } \\
\text { Ausgangslösung }\end{array}$ & $\begin{array}{l}\text { Vol. der Ausgangslösung in } \mathrm{ml} \\
\text { für } 1,75 \mathrm{~m} l \text { Ansatz }\end{array}$ \\
\hline $\begin{array}{l}\text { Adenosin-5'-diphosphat } \mathrm{C}_{10} \mathrm{H}_{12} \mathrm{O}_{10} \mathrm{~N}_{6} \mathrm{P}_{2} \mathrm{Na}_{3} \\
\text { Mol.-Gew. 493,2 } \\
\text { Boehringer ADP } 15014\end{array}$ & $4,5 \mathrm{~mm}$ & $\begin{array}{c}0,2219 \mathrm{~g} / 3,00 \mathrm{ml} \\
(150 \mathrm{~mm})\end{array}$ & 0,060 \\
\hline $\begin{array}{l}\text { MgSO } \cdot 7 \mathrm{H}_{2} \mathrm{O} \\
\text { Mol.-Gew. } 246,48 \\
\text { Merck } 5886\end{array}$ & $5,0 \mathrm{~mm}$ & $\begin{array}{l}0,2465 \mathrm{~g} / 10,00 \mathrm{ml} \\
(100 \mathrm{~mm})\end{array}$ & 0,100 \\
\hline $\begin{array}{l}\text { Nicotinamid-adenin-dinucleotid, red. } \\
\mathrm{C}_{2}, \mathrm{H}_{2}, \mathrm{O}_{1}, \mathrm{~N}_{2} \mathrm{P}_{2} \mathrm{Na}_{2} \\
\mathrm{Mol}_{1}-\mathrm{Gew}, 709,4 \\
\text { Boehringer NADH } 15142\end{array}$ & $0,30 \mathrm{~mm}$ & $\begin{array}{c}0,0213 \mathrm{~g} / 2,00 \mathrm{ml} \\
(15,0 \mathrm{mM})\end{array}$ & $0,040^{\circ}$ \\
\hline $\begin{array}{l}\text { 1.1.27 L-Lactat: NAD-Oxidoreductase } \\
\text { Boehringer LDH-M-R 15371 }\end{array}$ & $38 \mu \mathrm{Mol} /(\operatorname{Min} . \cdot \mathrm{ml})$ & & $\mathrm{V}_{\text {LDH }}$ \\
\hline $\begin{array}{l}\text { Pufferlösung pH }=7,6 \\
\text { Triäthanolamin- } \mathrm{HCl} \mathrm{C}_{8} \mathrm{H}_{18} \mathrm{O}_{3} \mathrm{~N} \cdot \mathrm{HCl} \\
\text { Mol.-Gew. 185,7 } \\
\text { Boehringer TRA } 15325 \\
\mathrm{NaOH} 2 \mathrm{M} \text {, Merck } 9136\end{array}$ & & $\begin{array}{l}9,285 \mathrm{~g} \text { TRA in } 90 \mathrm{ml} \text { Aqua } \\
\text { tridest. lösen, } 6,52 \mathrm{ml} 2 \mathrm{M} \mathrm{NaOH} \\
\text { hinzufügen und ad } 100,0 \mathrm{ml} \\
\text { mit Aqua tridest. auffüllen. } \\
\text { (,,TRA" } 500 \mathrm{mM}, \mathrm{NaOH} 134 \mathrm{~mm} \text { ) }\end{array}$ & $\mathrm{V}_{\mathbf{P}}$ \\
\hline
\end{tabular}

Wenn die Meßtemperatur erreicht ist, werden $0,050 \mathrm{ml}$ der zu testenden Lösung (10-2 Verdünnung der Enzym-Suspension, die unmittelbar vor Wenn die Meßtemperatur erreicht ist, werden $0,050 \mathrm{ml}$ der zu testenden Lösung (10-2 Verdünnung der Enzym-Suspension, die unmittelbar vor der Messung hergestellt werden muß) hinzugefügt und
$20 \mathrm{mM}: 0,0207 \mathrm{~g}$ mit Pufferlösung ad $5,00 \mathrm{ml}$ ) gestartet. 
zelnen Testansätze ermittelt wurde. Wenn man die Aktivität einer CPK-Lösung mit verschiedenen Verfahren bestimmt, erhält man unterschiedliche Werte (Tabelle 4 und (4)).

Im „Report of the commision on enzymes of the International Union of Biochemistry" (6), wird als Enzymeinheit diejenige Menge definiert, die ein Mikromol Substrat in der Minute unter definierten experimentellen Bedingungen (standard conditions) umsetzt. Dieser Report empfiehlt eine Meßtemperatur von $30^{\circ}$ und begründet diese Empfehlung damit, daß es ein großer Vorteil wäre, eine Standardtemperatur zu haben, um so einen Vergleich zwvischen den Aktivitäten der verschiedenen Enzyme zu ermöglichen. Die übrigen Bedingungen müßten, wenn möglich, optimal sein, insbesondere $\mathrm{pH}$ und Substratkonzentration. Der Report begründet nicht, warum nicht auch für die Temperatur optimale Werte ermittelt werden müßten, und warum eine gleiche Temperatur bei der Messung der verschiedenen Enzymaktivitäten erforderlich ist, um einen Vergleich zwischen diesen Aktivitäten zu ermöglichen.

Unserem Meßverfahren liegen de folgenden Reaktionen zugrunde (7):

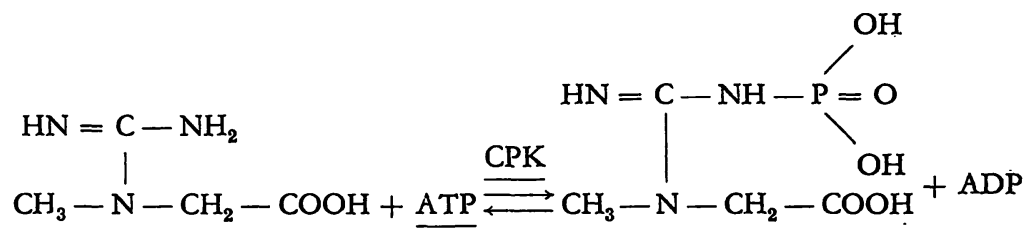

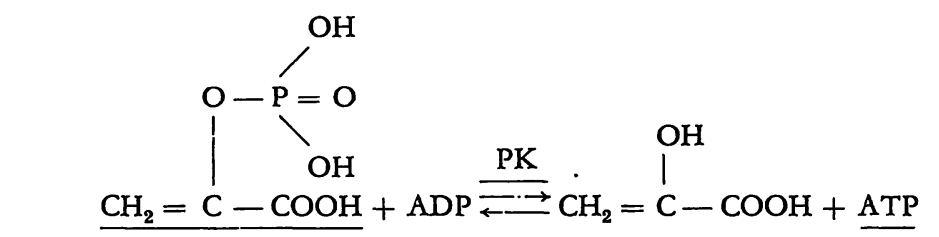

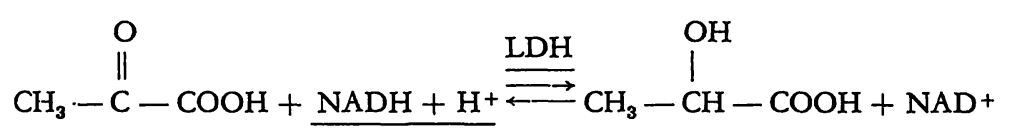
tional.
Küvette nach der folgenden Gleichung berechnet (Gesetz von Lambert und BeER):

$$
\frac{d c}{d t}=\frac{d E}{d t \cdot \varepsilon \cdot s}
$$

Die so gemessene Aktivität ist der eingesetzten Menge kristalliner CPK über einen weiten Bereich propor-

Wenn die spezifische Aktivität des Enzym-Kristallisats in der Küvette im Bereich von 0,06 bis 0,004 $\frac{\mu \mathrm{Mol}}{\mathrm{Min} \cdot \mathrm{m} l}$ liegt, dann ist die gemessene Aktivität der Enzym-Konzentration proportional. Wegen der schnell eintretenden Inaktivierung reiner Enzym-Lösungen bei hoher Verdünnung ist es nicht möglich, bei geringeren spezifischen Aktivitäten genaue Ergebnisse zu ermitteln.

Bei Einsatz von menschlichem Serum besteht Proportionalität zwischen Enzym-Konzentration in der Küvette und gemessener Aktivität im Bereich von 0,005 bis $0,0001 \frac{\mu \mathrm{Mol}}{\text { Min. ml }}$.

Beim Vergleich unseres Testansatzes mit dem von RotTHAUwe und Mitarbeiter (3) angegebenen und mit dem der $\mathrm{Fa}$. Boehringer ergaben sich folgende Unterschiede: Der erstgenannte wird nach einem $\Delta \mathrm{E}$ von 0,05 linear und bleibt es über ein $\Delta \mathrm{E}$ von 0,20. Bei dem Boehringer-Rest bleibt die Reaktionsgeschwindigkeit nach einem $\Delta \mathrm{E}$ von 0,10 während eines. $\Delta \mathrm{E}$ von 0,15 konstant. Obwohl bei unserem Test der lineare $\mathrm{Ab}$ lauf erst nach einer Extinktionsänderung von 0,20 erreicht wird, beginnt die Reaktion wegen ihrer höheren Geschwindig-

Die Auslösung der Reaktion erfolgt durch Zugabe von $\alpha$-Methylguanidinessigsäure (Kreatin) zu einer Lösung, die alle im obigen Reaktionsschema unterstrichenen Substanzen enthält. Nach einer gewissen Zeit erreicht die Umsatzgeschwindigkeit von $\mathrm{NADH}$ diejenige von Kreatin.

Unter optimalen Bedingungen erfolgt die Aktivitätsbestimmung bei $38,0-40,0^{\circ}$ durch photometrische Messung der Extinktionsänderung bei $366 \mathrm{~nm}$. Wir benutzten das Photometer „Eppendorf" sowie Küvetten der Firma Netheler \& Hinz, Hamburg. Ein thermostabilisierter Küvettenhalter hielt die Temperatur auf $(38,0 \pm 0,1)^{\circ}$. Das Photometer war mit einer Registriereinrichtung der Firma Philips verbunden, die eine graphische Darstellung der Extinktion als Funktion der Zeit aufzeichnet.

Bei Benutzung einer Küvette der Schichtdicke 1,000 cm und Einsatz kristalliner CPK für die Messung nimmt die Geschwindigkeit der Extinktionsänderung bis zu einem $\Delta \mathrm{E}$ von 0,20 noch $z u$, um dann während eines $\Delta \mathrm{E}$ von 0,50 konstant zu bleiben. Danach tritt wieder eine Abnahme der Reaktionsgeschwindigkeit ein.

Unter Zugrundelegung des linearen Teiles der ZeitExtinktions-Kurve wird die Enzymaktivität in der keit erheblich früher linear zu laufen als bei dem der Fa. Boehringer und nur wenig später als bei RotrHAUWE.

Der große Linearitätsbereich ist besonders wichtig bei der Verwendung von Mikroküvetten, da man bei diesen mit erhöhter Verstärkung arbeiten muß, und deshalb durch die Instabilität des Gerätes bedingte Abweichungen vom linearen Verlauf auftreten können.

Tab. 4
Vergleich unseres Testes mit verschiedenen anderen

\begin{tabular}{|c|c|c|}
\hline & $\begin{array}{c}\text { Geschwindigkeit } \\
\text { der Teste auf die } \\
\text { Geschwindigkeit } \\
\text { unseres Testes bei } \\
(38,0 \pm 0,1)^{\circ} \\
\text { bezogen }\end{array}$ & $\begin{array}{c}\text { Mit den Testen } \\
\text { gemessene Aktivität } \\
\text { auf die mit unserem } \\
\text { Test gemessene } \\
\text { bezogen }\end{array}$ \\
\hline $\begin{array}{l}\text { Unser Test }(38,0 \pm 0,1)^{\circ} \\
0,15 \mathrm{ml} \text { Serum, } \\
0,50 \mathrm{ml} \text { Gesamtvolumen }\end{array}$ & 1,0 & 1,0 \\
\hline $\begin{array}{l}\text { Unser Test }(25,0 \pm 0,1)^{\circ} \\
0,15 \mathrm{ml} \text { Serum, } \\
0,50 \mathrm{ml} \text { Gesa mtvolumen }\end{array}$ & 0,49 & 0,49 \\
\hline $\begin{array}{l}\text { Boehringer-Test }(38,0 \pm 0,1)^{\circ} \\
1,00 \mathrm{~m} l \text { Serum, } \\
3,50 \mathrm{ml} \text { Gesamtvolumen }\end{array}$ & 0,40 & 0,42 \\
\hline $\begin{array}{l}\text { Boehringer-Test }(25,0 \pm 0,1)^{\circ} \\
1,00 \mathrm{ml} \text { Serum, } \\
3,50 \mathrm{ml} \text { Gesamtvolumen }\end{array}$ & 0,17 & 0,18 \\
\hline $\begin{array}{l}\text { Rotthauwe-Test }(37,0 \pm 0,1)^{\circ} \\
0,50 \mathrm{ml} \text { Serum, } \\
2,00 \mathrm{ml} \text { Gesamtvolumen }\end{array}$ & 0,30 & 0,36 \\
\hline
\end{tabular}


Da bei Einsatz von biologischem Untersuchungsmaterial das NADH des Testansatzes auch ohne Zugabe von Kreatin umgesetzt wird, ist es erforderlich, eine Aktivitätsbestimmung ohne Kreatin (Vorlauf) durchzuführen und die dabei gemessene Aktivität von der mit Kreatin gemessenen (Hauptlauf) abzuziehen. $\mathrm{Da}$ die Linearität des Vorlaufs oft erst jenseits eines gewissen $\Delta \mathrm{E}$ erreicht wird, muß der Testansatz eine ausreichende Menge von $\mathrm{NADH}$ enthalten. Dieses $\triangle \mathrm{E}$ hängt von der Art des Untersuchungsmaterials $a b$ und ist daher von Fall zu Fall verschieden.

Die Auswirkung der verschiedenen Reaktionsparameter auf die gemessene CPK-Aktivität wird in den Abbildungen 1 bis 8 dargestellt.

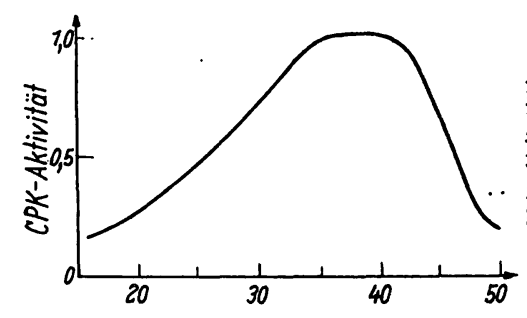

Abb. 1

CPK-Aktivität, bezogen auf die Aktivität bei $38^{\circ}$, als Funktion der Temperatur. Die Messungen erfolgten mit dem in Tabelle 1 angegebenen Verfahren

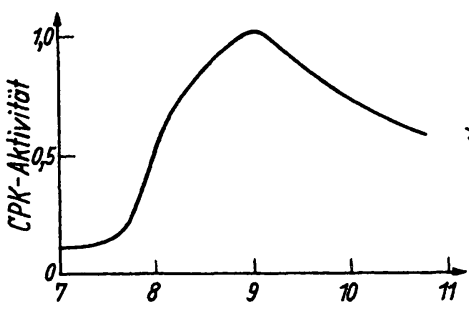

Abb. 2

CPK-Aktivität, bezogen auf die Aktivität bei $\mathrm{pH}=9,0$ als Funktion des $\mathrm{pH}$. Die Messungen erfolgten mit dem in Tabelle 1 angegebenen Verfahren. Das $\mathrm{pH}$ der Pufferlösung wurde durch Zugabe von $\mathrm{HCl}$ auf die verschiedenen Werte eingestellt

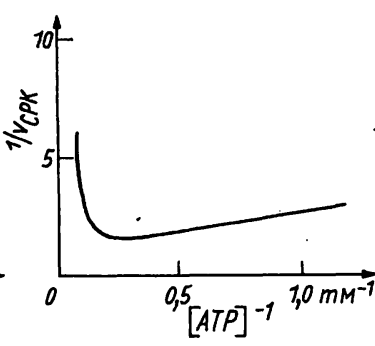

Abb. 3

Bestimmung der optimalen ATP-Konzentration im Testansatz. Die Konzentrationen der anderen Reaktionsteilnehmer sind in Tabelle 1 angegeben. Dieses gilt auch für die folgenden Abbildungen

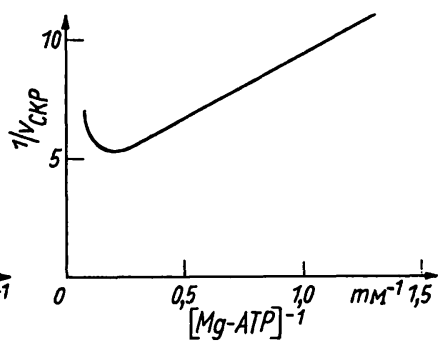

Abb. 4

Bestimmung der optimalen Mg-ATP Konzentration im Testansatz

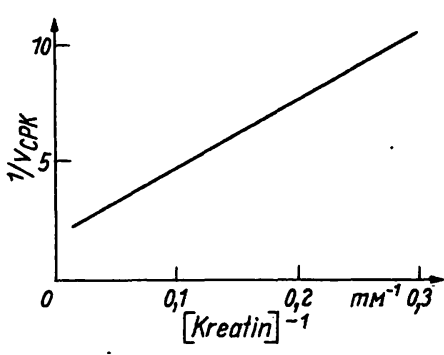

Abb. 5

Bestimmung der optimalen Kreatin-Konzentration im Testansatz. Wie man sieht, ergibt eine weitere Steigerung der Kreation-Konzentration nur eine geringe Erhöhung der Reaktionsgeschwindigkeit. Wegen der schlechten Löslichkeit des Kreatins ist eine Konzentration von $75 \mathrm{mM}$ praktisch die höchst erreichbare

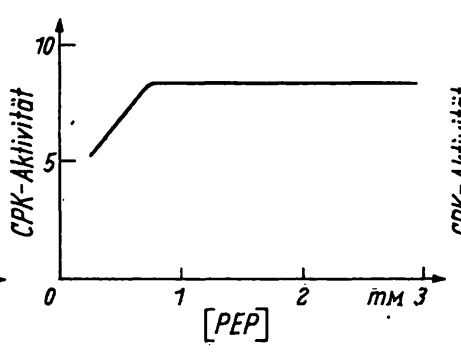

Abb. 6

Bestimmung der optimalen PEP-Konzentration im Testansatz

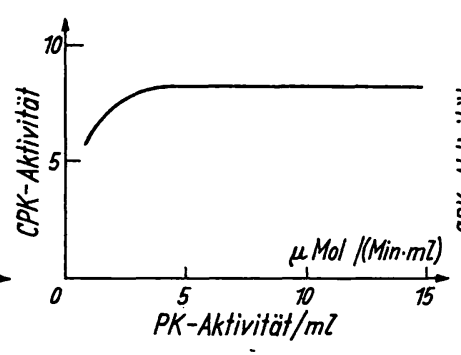

Abb. 7

Bestimmung der optimalen PKAktivität im Testansatz

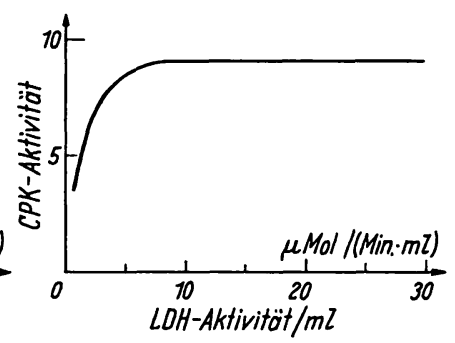

Abb. 8

Bestimmung der optimalen LDH-Aktivität im Testansatz

\section{Literatur}

1. CPK-Test, Biochemica, Boehringer, Mannheim (1966). - 2. Cotombo, J. P., R. Richterich und E. Rossi, Klin. Wschr. 40, 37 (1962). - 3. Rotthauwe, H. W., S. Zurukzoglu-SkLavounou und H. HammanN, Klin. Wschr. 39, 1269 (1961). - 4. Rotthauwe, H. W. und M. Cerquerro-Rodriguez, Clin. chim. Acta (Amster- dam) 10, 134 (1964). - 5. KLEINE, T. O., Klin. Wschr. 42, 504 (1965). - 6. Frorkin, M. und E. H. Stotz (Hrsg.), Comprehensive Biochemistry, Bd. 13, Elsevier Publ., Amsterdam-London-New York (1964). - 7. Tanzer, M. L. und Cir. Gilvarg, J. biol. Chemistry 234, 3201 (1959).

Dr. M. Feraudi

2 Hamburg 20, Martinistr. 52 